\title{
Non-Linear Responses and Surprises
}

We certainly live in a nonlinear world where rapid and unpredicted system responses can result from small changes in forcing conditions or gradual and continuous environmental change. Nonlinear behavior may emerge from complex interactions and feedbacks or from simple biochemical and structural threshold-like responses with cascade effects throughout the functioning of a system. Such a behavior can display from deterministic chaos to emergent complexity and self-organization that, for instances, can result in multiple steady states for a given system.

This type of behavior is very much part of the way ecological systems function, yet, tremendous efforts with numerical and experimental approaches have been geared to simplify complex nonlinear responses into a more easily tractable linear world. The linear thinking is also much entrenched in the way policy perceives environmental change and, in consequence, ways to manage it (e.g. the assumption that climate change will alter production of terrestrial ecosystems progressively).

A deeper understanding of the nonlinear nature of systems will contribute to increasing our capacity to predict future ecosystem behavior under novel combinations of resources and forcings that brought about global change, and to detect early in advance low-probability catastrophe events, such as the terrestrial biosphere's flip from $C$ sink to C source or the collapse of production systems.

IGBP, building upon an early initiative from GCTE, has initiated a new research focus to study biospheric responses to global change that involve rapid nonlinear changes and thresholds. The initiative is very much in its early stages of development but some of the emerging objectives are:

- To identify processes and resource gradients more prone to generate nonlinear responses, and therefore, unexpected system's behavior under future global change.

- To understand when nonlinear responses are important as related to scaling issues in time and space.

- To quantify transfer functions between forcings and responses for verifying and parameterizing predictive process models.

- To develop new numerical tools and experiments to study nonlinear responses (e.g., thresholds).

- To formulate new hypotheses derived from mathematical treat- ment of model systems (e.g., spatial scale-dependent effects of perturbations) that may be tested using reconstruction methods.

- To use knowledge of nonlinear phenomena to better guide policy development for adaptation strategies and mitigation.

A scientific committee has been assembled to prepare a first workshop that will bring together relevant research on this field from various core projects of IGBP. The committee is made up of Ian Noble (GCTE), Jim Reynolds (GCTE), John Dearing (PAGES), John Schellnhuber (GAIM), Paul Crutzen (IGBPSSC, IGAC), Roger Pielke (BAHC), TBA (JGOFS), and Pep Canadell (coordinator). Inquires about this new activity can be sent to Pep Canadell (pep.canadell@gcte.org). This Duke University meeting is a further stage in IGBP nonlinear initiatives, and, in PAGES terms, partly builds on an earlier article in the PAGES Newsletter 99-2. PAGES Newsletter readers wishing to have an input to this initiative should contact me - all views will be most welcome.

\section{John A. Dearing}

Department of Geography, University of Liverpool, UK i.dearing@liverpool.ac.uk

\section{Land Use and Climate Impacts on Fluvial Systems During the Period of Agriculture (LUCIFS)}

LUCIFS aims to understand the variations in water and particulate fluxes through fluvial systems at various times since agriculture began on our planet. We wish to know how fluvial systems have responded to past changes in climate and/or land-use, what factors controlled fluxes of water and particulates (sediment, particulate nutrients and carbon), how sensitivity to these factors varies in space and time, and how present day changes are affected by long-term processes and trends. Finally, LUCIFS wishes to contribute to our understanding of the feedbacks to global environmental change from changes in fluvial systems. Such feedbacks occur principally through changes in the carbon cycle, modulated by sediments and nutrients delivered to the coastal zone by rivers.

There are currently 20 case studies in LUCIFS, from New Zealand, the Pacific, Europe, Asia, Australia, and the Americas. These case studies are being analyzed within conceptual frameworks that are general enough to cope with a wide range of system types. Material budgets are central to all LUCIFS case studies. In addition, one framework that is being used and further explored consists of input time series (eg. land use and climate change) and output series (eg. sedimentation and nutrient fluxes to sinks such as lakes and reservoirs), linked by transfer functions that modulate inputs to produce outputs. This system - level understanding is essential to meet the aims of LUCIFS.

Further information about LUCIFS can be found at: http://www.fas.nus. edu.sg/geog/davidt.htm.

\section{BOB WAsSON}

Australian National University, Canberra, Australia robert.wasson@anu.edu.au

\section{Aleksey Sidorchuk}

State University of Moscow, Moscow, Russia yas@yas.geogr.msu.su

\section{Reference}

Wasson, R.J. (ed.) Land Use and Climate Impacts on Fluvial Systems during the Period of Agriculture. PAGES Workshop Report, Series 96-2, 1996. $51 \mathrm{pp}$. 